\title{
Archaeonautica
}

Archaeonautica

L'archéologie maritime et navale de la préhistoire à

l'époque contemporaine

$20 \mid 2018$

De re navali : Pérégrinations nautiques entre Méditerranée et océan Indien

\section{De re navali : Pérégrinations nautiques entre Méditerranée et océan Indien. Mélanges en l'honneur de Patrice Pomey}

\section{Giulia Boetto et Éric Rieth}

\section{(2) OpenEdition}

\section{Journals}

Édition électronique

URL : http://journals.openedition.org/archaeonautica/382

DOI : 10.4000/archaeonautica.382

ISSN : 2117-6973

Éditeur

CNRS Éditions

\section{Édition imprimée}

Date de publication : 6 décembre 2018

Pagination : 7-15

ISBN : 978-2-271-12263-6

ISSN : 0154-1854

Référence électronique

Giulia Boetto et Éric Rieth, « De re navali : Pérégrinations nautiques entre Méditerranée et océan Indien. Mélanges en l'honneur de Patrice Pomey », Archaeonautica [En ligne], 20 | 2018, mis en ligne le 30 avril 2020, consulté le 15 septembre 2020. URL : http://journals.openedition.org/archaeonautica/382 


\title{
De re navali: PéréGrinations nautiques entre MéditerRanée et ocÉan InDien. Mélanges en l'honneur de Patrice Pomey
}

\author{
Giulia Boetto, Eric Rieth
}

Très attaché à Paris, sa ville natale et aux quartiers de sa jeunesse, entre boulevard Saint-Germain et place EdmondRostand, Patrice Pomey est devenu depuis les années 1975 un provençal d'adoption renouant avec une part de ses origines familiales. Une fois son baccalauréat obtenu, il hésita un temps sur le choix de ses études supérieures. Allait-il s'orienter vers une formation scientifique ? Allait-il au contraire se tourner vers l'univers des arts en s'inscrivant ainsi dans une autre tradition familiale ? C'est une troisième voie qu'il choisit de suivre en optant, après une classe préparatoire scientifique ouvrant le chemin vers les concours aux grandes écoles d'ingénieur, pour des études d'archéologie à la Sorbonne.

De 1966 à 1970, Patrice fréquenta l'amphithéâtre aménagé en sous-sol et les salles de cours, dont la fameuse salle des plâtres, de l'Institut d'histoire de l'art et d'archéologie de la Sorbonne situé rue Michelet, à côté du Petit Luxembourg. Dans les années 1970, il eut la chance de suivre les cours de grands noms de l'archéologique classique et du Proche-Orient tels Jean Deshayes, Pierre Demargne ou encore Gilbert-Charles Picard. C'est sous la direction de ce dernier, professeur d'archéologie classique à l'université de Paris-Sorbonne, que Patrice a soutenu brillamment en 1970 son mémoire de maîtrise intitulé Recherches sur la construction navale à l'époque romaine. Il fréquenta également le séminaire animé par Paul-Marie Duval dans le cadre de la IV ${ }^{\mathrm{e}}$ section de l'École Pratique des Hautes Études. Si l'archéologie gallo-romaine (l'histoire antique de Paris notamment) constituait le thème central des recherches de P.-M. Duval, celui-ci n'était pas insensible aux questions maritimes comme le montre son étude classique de la mosaïque d'Althiburus. À la même époque et dans le même cadre de l'EPHE, Patrice suivit également le séminaire d'histoire maritime médiévale et moderne de Michel Mollat du Jourdin, professeur à la Sorbonne, et qui fut à l'origine du renouveau en France des études d'histoire maritime. C'est sous sa direction qu'il présenta en 1971 à l'EPHE un mémoire consacré à une Étude des navires figurés sur les mosaïques de la place des Corporations à Ostie.

Après un court passage dans l'enseignement supérieur (19701971) comme assistant en histoire de l'art et archéologie de l'Antiquité à l'université de Nancy 2, Patrice intégra l'École française de Rome. Ces « années romaines », de 1971 à 1974, jouèrent un rôle fondamental dans la formation scientifique de Patrice. C'est en effet lors de son séjour à l'EFR qu'il trouva le sujet de sa thèse de doctorat de $3^{\text {e }}$ cycle sur l'iconographie des «naves onerariae » d'Ostie, dirigée par le Pr Gilbert-Charles Picard et brillamment soutenue en 1974. Si, pour des raisons médicales, Jean Rougé, le grand historien du commerce maritime de l'Empire romain, n'eut pas la possibilité de participer au jury, il suivit régulièrement, en revanche, l'avancement de la thèse et, par la suite, les recherches de Patrice.

C'est également pendant son séjour à l'EFR, que Patrice rencontra le jeune archéologue italien Piero Alfredo Gianfrotta avec lequel il rédigea l'ouvrage Archeologia subacquea publié dans sa version originale italienne en 1981. Enfin, comment ne pas évoquer la rencontre entre Patrice, l'archéologue, et Monique, la restauratrice spécialiste en peintures alors étudiante à l'Istituto Centrale del Restauro, et imaginer, pourquoi pas, que l'esprit de la fontaine de Trevi ou la magie de la ville éternelle ne furent peut-être pas étrangers à cette rencontre!

De retour en France, Patrice intégra le CNRS au sein duquel, d'abord dans le cadre aixois de l'Institut d'archéologie méditerranéenne, puis dans celui du centre Camille-Jullian, il effectua toute sa brillante carrière de chercheur qu'il termina comme directeur de recherche. Après avoir soutenu en 1997 son Habilitation à Diriger des Recherches à l'université de Provence, il dirigea ce même laboratoire de 2000 à 2007. Ce choix de s'investir dans des responsabilités collectives représente une autre facette de sa personnalité dont la politique, en ce domaine, a toujours été de faire des choix administratifs en fonction de projets scientifiques. C'est bien en effet cet intérêt qui guidait ses décisions lorsque, de 1984 à 1991, il assura les fonctions de directeur de la Direction des Recherches Archéologiques SousMarines du ministère de la Culture. Déployant une intense activité d'administration du patrimoine archéologique sous-marin, effectuant de multiples visites de chantiers de fouilles sous-marines le long des côtes de la Méditerranée et de l'Atlantique, renouant des liens parfois quelque peu distendus avec les archéologues bénévoles, il fut porteur de la loi de 1989 sur les biens culturels maritimes qui régit aujourd'hui encore l'archéologie sous-marine française.

On pourrait s'étendre longuement sur le parcours scientifique de Patrice. Nous voudrions nous arrêter sur quelques aspects, parmi les plus représentatifs, de sa carrière de chercheur. Nous avons déjà évoqué le livre Archeologia subacquea publié avec P. A. Gianfrotta. Dans les pages relatives à l'histoire de l'architecture navale antique dont Patrice est l'auteur, se perçoit la qualité de son raisonnement, la précision de ses arguments, la rigueur de ses analyses, la logique de ses interprétations, la prudence de ses conclusions. Cet « esprit de la méthode Pomey » que l'on retrouve dans ses publications est sans doute, pour une part tout au moins, la résultante d'une aptitude et d'une ouverture intellectuelle aux matières scientifiques renvoyant à ses études secondaires et aux interrogations face aux choix de sa formation universitaire. 
Dans le parcours scientifique de Patrice, une rencontre allait être déterminante : celle avec André Tchernia en 1967 alors que celui-ci était assistant de latin à l'université de Provence et premier directeur de la Drasm. Pour le littéraire qu'était A. Tchernia, l'archéologie navale relevait de problématiques historiques où la dimension technique occupait une place essentielle. C'est également lui qui introduisit Patrice dans la communauté des archéologues-plongeurs en lui confiant l'étude architecturale de l'épave Planier 3 et l'associant, comme codirecteur avec Antoinette Hesnard, à la fouille de l'épave de la Madrague de Giens de 1972 à 1982. Cette fouille de grande ampleur à laquelle collaborèrent avec talent les photographes Gérard Réveillac et Antoine Chéné et le dessinateur, Michel Rival, tous les trois membres du $\mathrm{CCJ}$, donna lieu à des expérimentations techniques et méthodologiques comme par exemple le marquage systématique des éléments de la coque et des assemblages, l'utilisation systématique de la photogrammétrie pour l'enregistrement de la cargaison et de la coque ou les prélèvements, à titre d'échantillons significatifs, d'éléments architecturaux. Une fois prélevés et remontés en surface, ces prélèvements « anatomiques », pour reprendre un terme de l'historien A. Jal considéré comme le « père » de l'archéologie navale, étaient démontés, observés, dessinés, étudiés d'une façon détaillée. La fouille de l'épave de la Madrague de Giens ne fut pas seulement un « laboratoire technique ». Ce fut également un exceptionnel « laboratoire scientifique » au cours duquel de nouveaux questionnements archéologiques furent élaborés sur, par exemple, la fonction, structurale ou conceptuelle, des varangues brochées, l'interprétation du sens de chevillage des tenons du bordé (distinction entre mise en place primaire à la construction et secondaire lors d'une réparation)... Ces questionnements ont toujours reposé sur des observations systématiques, répétées, recoupées, selon une méthode d'étude aussi rigoureuse que prudente.

D'une fouille sous-marine et terrestre à une autre, les recherches de terrain de Patrice contribuèrent à renouveler dans une large mesure l'histoire de l'architecture navale antique méditerranéenne. Sa bibliographie est le plus éloquent témoignage de son apport à la science. Dans le cadre de cette introduction, nous ne pouvons évoquer que quelques grandes tendances de ses travaux, en commençant par mentionner celle liée aux approches conceptuelles. On citera les deux notions, méthodologiquement fondamentales, de principe de conception sur le double plan morphologique et structural, et de méthode (ou procédé) de construction, la première renvoyant, suivant la classification des historiens des techniques, au champ de la pensée technique et la seconde à celui de la pratique technique ; la définition de nouveaux types architecturaux tels ceux de «type hellénistique » et «type romain impérial »; les recherches conduites sur l'architecture navale cousue antique dont la relecture des vestiges de l'épave de Bon Porté a constitué la première étape et dont l'interprétation des épaves grecques archaïques Jules Verne 7 et 9, chacune avec leurs spécificités et leurs degrés d'évolution technique de leur assemblage, a représenté l'une des dernières séquences dans l'état actuel d'avancement des recherches de Patrice tout au moins. Comment ne pas mentionner, enfin, les études relatives aux processus de transition architecturale, par évolution ou rupture, phénomènes complexes car prenant en compte de multiples facteurs d'ordre non seulement technique, mais aussi économique, socioculturel, environnemental dont les sources archéologiques en particulier offrent souvent des données fragmentaires et difficiles à interpréter. Pensons, par exemple, aux travaux sur les processus de transition entre architecture cousue intégrale, architecture cousue « mixte », architecture par assemblage du bordé par tenons, mortaises, chevilles. Rappelons les études sur la transition entre construction de principe « sur bordé » et construction de principe «sur membrure », une thématique partagée au demeurant par les spécialistes de l'Antiquité et du Moyen Âge.

Si la majorité des recherches réalisées par Patrice a été circonscrite à la Méditerranée, une partie, toutefois, a porté sur l'espace nautique si particulier de la mer Rouge. Dans le cadre de son séjour de deux ans (2008-2010) au Centre d'Études Alexandrines dirigé par son ami Jean-Yves Empereur, Patrice a mené plusieurs campagnes de fouille terrestre sur le site d'Ayn Soukhna. Au fond d'une galerie creusée dans la montagne littorale, la fouille réalisée dans des conditions difficiles a mis au jour les vestiges carbonisés et en partie écrasés par des effondrements de rochers de pièces de charpente (bordé et membrures) appartenant à de grands navires de mer construits suivant le même principe d'assemblage cousu que celui de la célèbre barque fluviale de Chéops. C'est donc un pan méconnu de la culture nautique maritime de l'Égypte antique que cette fouille a ainsi mis en évidence.

Les épaves n'ont pas été les seules sources sollicitées par Patrice pour son écriture de l'histoire de l'architecture navale antique. L'iconographie a tenu une place non négligeable dans ses travaux. Son intérêt pour l'histoire de l'art et la peinture contemporaine notamment, comme le démontre son rôle au sein de la Fondation Vasarely, n'est peut-être pas totalement étranger à sa sensibilité de chercheur face aux documents iconographiques de l'Antiquité. Sa thèse de troisième cycle est de ce point de vue annonciatrice, par exemple, de son étude des relevés des graffiti navals de Délos opérés par le commandant Carlini ou de celle relative à la relecture du graffito de Cucuron qui, toutes les deux, à la rigueur de l'indispensable analyse critique interne du document, allient la précision du regard permettant d'identifier, toujours avec prudence, un détail difficilement lisible, le tout reposant sur une connaissance technique sans faille de l'architecture navale antique lui évitant tout anachronisme. Quant à l'étude des mosaïques, l'exemple le plus récent est fourni par celle de Kelenderis, datée de la fin du $v^{e}$ siècle, dont l'analyse a mis en évidence l'emploi de la voile latine dite « latine orientale » (avec une courte chute avant) sous une forme très élaborée avec une bande de ris supérieure disposée en oblique.

Un autre aspect novateur des recherches de Patrice sur l'iconographie est la méthode avec laquelle il établit un dialogue entre l'image et le document archéologique. La plus belle démonstration est en ce domaine la restitution du gréement du navire de la Madrague de Giens sur la base de la célèbre mosaïque des thermes de Thémétra à Sousse figurant un grand navire à trois mâts (dont un artemon de proue). L'argumentation rigoureuse repose sur la mise en correspondance graphique entre les emplantures, et aussi l'archipompe, du voilier de la mosaïque et les vestiges de l'épave de la Madrague de Giens.

En évoquant ce thème de la reconstitution architecturale, une autre facette de la carrière de Patrice vient immédiatement à l'esprit. C'est celle de l'apport du modélisme de recherche aux études d'archéologie navale. Avec la collaboration de Robert Roman, ancien charpentier de marine et membre du CCJ, il a en effet développé au sein de l'équipe d'archéologie navale qu'il a créée un vaste programme de recherche d'archéologie navale expérimentale à échelle réduite prenant appui sur le modélisme de recherche dans le prolongement des réalisations des pionniers en la matière, l'Américain Dick Steffy et le Danois Ole 
Crumlin-Pedersen. Aux « écoles américaine et danoise », chacune avec leurs pratiques particulières de maquettisme, vient désormais s'ajouter une «école aixoise »dont Patrice a été l'inspirateur.

De la maquette à échelle réduite à la reconstitution d'un bateau à échelle grandeur nature, il n'y a qu'un pas... ou plutôt qu'une... immense enjambée qu'il était bien tentant de franchir. C'est le programme d'archéologie navale expérimentale Prôtis qui s'est concrétisé par la construction en 2013 du Gyptis, la reconstitution de l'épave Jules Verne 9, puis par la navigation du voilier en rade de Marseille et le long du littoral des Bouchesdu-Rhône et au-delà même. Deux jeunes archéologues, Sabrina Marlier et Pierre Poveda, contribuèrent grandement à la réussite de ce programme d'archéologie navale expérimentale.

Bien d'autres aspects de l'œuvre scientifique de Patrice mériteraient d'être mis en lumière. C'est le cas des multiples relations entretenues avec les « grands noms » de l'archéologie navale antique méditerranéenne, Lucien Basch, Lionel Casson, Honor Frost, Yaacov Kahanov, Dick Steffy pour ne citer que les disparus, sans oublier Paul Adam, au nom moins connu mais cher au cœur de Patrice. Parmi les autres évocations qu'il eut fallu faire, il y a l'intérêt de Patrice pour l'ethno-archéologie des bateaux cousus de l'Inde ou son enseignement que de nombreux étudiants ont eu la chance de suivre et qui, d'élèves, sont devenus aujourd'hui en France et à l'étranger des acteurs à part entière de la recherche en archéologie navale antique méditerranéenne.

Il nous faut à présent conclure.

Cette introduction à l'ouvrage d'hommages en l'honneur de Patrice Pomey voudrait aussi témoigner de notre profonde affection pour l'homme et l'ami. Alors, quittant la forme imposée par l'exercice de l'introduction à un livre, nous souhaitons conclure ces pages par une note plus personnelle des signataires.

\section{Giulia.}

Cher Patrice, c'était l'année 1993 lors d'un colloque d'archéologie sous-marine à Gela, en Sicile. Tu venais présenter les découvertes d'épaves de la place Jules-Verne et moi, étudiante en archéologie à l'université de Turin, je rêvais d'apprendre à étudier une épave antique et, qui sait, de venir un jour en France me spécialiser dans cette branche très particulière de la recherche archéologique dont la «bible »était le "Gianfrotta, Pomey », cet ouvrage de référence qui a servi de guide à tant d'archéologues sous-marins en Italie comme dans bien d'autres pays. Il m'a fallu patienter, puis j'ai débarqué à Aix-en-Provence trois ans plus tard et depuis les allers-retours Italie-France ont cadencé mon parcours d'étude et de recherche tout comme nos rendez-vous pour le suivi de mon diplôme de spécialisation en archéologie à Turin et de mon travail de thèse en archéologie navale. Avoir pu bénéficier durant toutes ces années de ton enseignement, de ta direction et de tes conseils, ainsi que de ton amitié aussi dans les moments difficiles, a été une chance inouïe. C'est grâce à toi que je me suis construite en tant que chercheur et que mon regard sur la Méditerranée antique et ses bateaux s'est affiné. Les nouveaux projets ne manquent pas. Alors embarquons pour de nouvelles et belles navigations scientifiques!

Eric.

Bien cher Patrice, notre première rencontre, dans la salle des plâtres de Michelet me semble-t-il, remonte aux années post 68 si riches en idées, projets, réalisations, expériences, utopies aussi, mais les utopies ne sont-elles pas indispensables à la vie ? Cela fait donc presque cinquante ans que nous cheminons ensemble, "compagnons de route "selon une expression chère à nos amis d'alors hélas disparus Jacques, Patrice, Pierre-Jean et d'autres, cheminement professionnel comme chercheur au CNRS, toi en archéologie navale antique, moi en archéologie navale médiévale. Que d'heures passées à discuter face à face à Paris et à Aix-en-Provence ou au téléphone en occupant longuement la ligne. Que de jours vécus ensemble lors de colloques en France et à l'étranger, de voyages d'étude, de séminaires. Que de rencontres partagées avec Dick, Lucien, Ole, Paul, Yak, et bien d'autres amis et collègues de par le monde. Nos publications écrites en collaboration ne sont en vérité qu'un reflet bien incomplet de notre longue complicité intellectuelle. Mais au-delà de celle-ci, il y a ce qui reste l'essentiel, cette complicité personnelle forgée par ces presque cinquante années d'amitié.

\section{BIBLIOGRAPHIE DE PATRICE POMEY}

On ajoutera à cette liste les très nombreux comptes rendus qu'il a publiés dans des revues comme The International Journal of Nautical Archaeology, The Mariner's Mirror, etc. et les articles de vulgarisation dans des revues grand public comme Archéologia, Neptunia, etc.

La bibliographie publiée est arrêtée à mars 2018.

\section{Ouvrages}

1970 Recherches sur la construction navale à l'époque romaine, mémoire de maîtrise, Université de Paris-Sorbonne (Paris IV), sous la direction de G. C. Picard, Paris [non publié]. Étude iconographique des «naves onerariae » d'Ostie, thèse de doctorat, Université de Paris-Sorbonne (Paris IV), sous la direction de G. C. Picard, Paris, 2 vol. [non publiée]. (XXXIVe suppl. à Gallia) (avec A. Tchernia, A. Hesnard et alii).
1981 Archeologia Subacquea: storia, tecniche, scoperte e relitti, Milan, Arnoldo Mondadori ; édition française (1981), L'archéologie sous la mer : histoire, techniques, découvertes et épaves, Paris, F. Nathan (avec P. A. Gianfrotta).

1997 Archéologie navale antique, habilitation à diriger des recherches, Université de Provence, sous la direction de A. Tchernia, 3 vol., Aix-en-Provence [non publié].

1997 La navigation dans l'Antiquité, Aix-en-Provence, Édisud (direction, avec P. A. Gianfrotta, X. Nieto et A. Tchernia).

1998 Construction navale maritime et fluviale. Approches archéologique, historique et ethnologique, Actes du $7^{\text {e International }}$ Symposium on Boat and Ship Archaeology (ISBSA 7), Île Tatihou, 1994, Paris, CNRS Éditions (Archaeonautica, 14) (dir. avec É. Rieth).

2002 Les Étrusques en mer : épaves d'Antibes à Marseille, catalogue de l'exposition, Aix-en-Provence, Musée de Marseille/Édisud (dir. avec L. Long et J.-Ch. Sourisseau). 

(avec É. Rieth).

2008 L'archéologie maritime et navale de la préhistoire à l'époque contemporaine, Archaeonautica, 15 (2009), Paris, éditions du CNRS (éd.).

2009 Transferts technologiques en architecture navale méditerranéenne de l'Antiquité aux Temps modernes : identité technique et identité culturelle, Actes de la Table Ronde Internationale, Istanbul, 2007, Istanbul, IFEA (Varia Anatolica, XX) (dir.).

2011 Batellerie Gallo-Romaine, Pratiques régionales et influences maritimes méditerranéennes, Paris/Aix-en-Provence, Éditions Errance/Centre Camille-Jullian (BiAMA, 9) (dir. avec G. Boetto et A. Tchernia)

2014 Ports et navires dans l'Antiquité à l'époque byzantine, Dossiers d'Archéologie, 364, juillet/août (éd.).

2015 La batellerie égyptienne. Archéologie, histoire, ethnographie, Alexandrie, Centre d'Études Alexandrines (Études Alexandrines, 34) (éd.).

\section{Articles et contributions à des ouvrages}

1969 L'archéologie sous-marine, Gazette des Beaux-Arts, p. 105-116. 1971 Autopsie d'une épave, Connaissance de la mer, 8, p. 80-85 (avec A. Tchernia).

1971 Expérience de détection magnétique sous-marine d'une épave antique, Prospezioni Archeologiche, 6, p. 65-71 (avec Y. Chevalier et A. Hesse).

1973 L'architecture navale romaine et les fouilles sous-marines, dans P.-M. Duval (éd.), Recherches d'archéologie celtique et galloromaine, III, 5, Paris-Genève, Droz, p. 37-51.

1973 Plaute et Ovide architectes navals !, MEFRA, 85, 2, p. 483-515.

1978 Le tonnage maximum des navires de commerce romains, Archaeonautica, 2, p. 233-251 (avec A. Tchernia).

1978 Les navires de commerce romains, Les Dossiers de l'Archéologie, 29, p. 20-29.

1981 L'épave de Bon-Porté et les bateaux cousus de Méditerranée, The Mariner's Mirror, 67, 3, p. 225-244.

1982 Le navire romain de la Madrague de Giens, CRAI, janvier-mars, p. 133-154.

1983 Remarques à propos de l'éperon d'Athlit, The Mariner's Mirror, 69,3 , p. $247-248$

1983 Archéologie navale antique, Universalia 1983, Suppl. апnиel Encyclopaedia Universalis, Paris, Albin Michel, p. 403-406.

1984 Archéologie sous-marine, dans Encyclopaedia Universalis, Paris, Encyclopaedia Universalis, p. 525-532 (avec A. Tchernia).

1984 Le rôle et l'intérêt des maquettes dans les études d'archéologie navale antique, Revue de la fondation océanographique Ricard, 7, p. $75-79$.

1985 Informations Archéologiques ; Recherches sous-marines, Gallia, 43, p. 547-576 (avec B. Liou).

1985 Perspectives d'avenir pour l'archéologie sous-marine : l'archéologie en eau profonde, dans $2^{e}$ Journées d'étude sur la plongée scientifique, Bulletin de l'Institut Océanographique de Monaco, n. spécial, 4, p. 59-74

1985 Mediterranean Sewn Boats in Antiquity, dans S. McGrail, E. Kentley (eds), Sewn Plank Boats, Oxford, British Archaeological Reports (BAR, Int. Series, 276), p. 35-47.

1987-1988 Recherches sous-marines, Gallia Informations, 1, p. 1-78 (dir., avec L. Long, M. L'Hour et Fl. Richez).

1988 Principes et méthodes de construction en architecture navale antique, dans Navires et commerces de la Méditerranée antique, Mélanges J. Rougé, Lyon, Comité historique du Centre-Est (Cahiers d'Histoire, XXXIII, 3-4), p. 397-412.

1991 Besoins en technologie de l'archéologie sous-marine, dans Technologies sous-marines pour la recherche et le développement, Actes du colloque IFREMER, n. 12 (avec A. Tchernia).

1991 La protection du patrimoine archéologique sous-marin : le cas de Marseille, Marseille, Atelier du Patrimoine, documents techniques, III.

1991 Épaves du Moyen Âge et de la Renaissance sur les côtes françaises, dans Ch. Villain-Gandossi, S. Busutti, P. Adam (eds), Medieval ships and the birth of technological societies, vol. II:
The Mediterranean area and European integration, La Valette, Fundation for International Studies, University of Malta, p. 437-445.

1991 La construction à franc-bord dans l'Antiquité méditerranéenne : principe et méthodes, dans R. Reinders, K. Paul (eds), Carvel Construction Technique, Proceedings of the Fifth International Symposium on Boat and Ship Archaeology (ISBSA 5), Amsterdam, 1998, Oxford, Oxbow Books (Oxbow Monograph, 12), p. 10.

1992 Recherches sous-marines, Gallia Informations, 1 (1993), p. 1-85 (avec L. Long, M. L’Hour, H. Bernard et Fl. Richez).

1992 Les premiers échanges maritimes du Midi de la Gaule du $\mathrm{VI}^{\mathrm{e}}$ au III e s. av. J.-C. à travers les épaves, dans M. Bats, G. Bertucchi, G. Congès, H. Tréziny (dir.), Marseille grecque et la Gaule, Lattes/Aix-en-Provence, ADAM/PUP (Études Massaliètes, 3), p. 189-198.

1993 Le patrimoine archéologique maritime de l'Antiquité, dans Le patrimoine maritime et fluvial, Actes du Colloque Estuaire 92, Nantes, 1992, Paris, Direction du Patrimoine, Ministère de la Culture, p. 22-27.

1993 Archéologie sous-marine, dans Encyclopédie de la Plongée, Paris, Vigot, p. 57-74.

1993 Le navire de Cucuron : un graffito décoratif, Archaeonautica, 11, p. 149-163.

1993 Bouches-du-Rhône. Au large de Marseille. Prospection thématique, dans Bilan scientifique DRASM 1992, Paris, Ministère de l'Éducation Nationale et de la Culture, p. 44.

1993 Les épaves romaines et grecques, dans Le Temps des découvertes. Marseille de Prôtis à la reine Jeanne, catalogue de l'exposition, Marseille, Musées de Marseille, p. 59-62 (avec A. Hesnard)

1993 Marseille. Place Jules-Verne. V - Les épaves romaines et grecques, dans Bilan scientifique DRAC-Provence-Alpes-Côte d'Azur, Paris, Ministère de la Culture et de la Francophonie, p. 112-115 (avec A. Hesnard).

1994 Shell Conception and Skeleton Process in Ancient Mediterranean Shipbuilding, dans Ch. Westerdahl (ed.), Crossroads in Ancient Shipbuilding, Proceedings of the Sixth International Symposium on Boat and Ship Archaeology (ISBSA 6), Roskilde, 1991, Oxford, Oxbow Books, p. 125-130 (Oxbow Monograph, 40).

1994 Var. Au large de Hyères. Prospection thématique, dans Bilan scientifique DRASM 1993, Paris, Ministère de la Culture et de la Francophonie, p. 43-44.

1995 Les épaves grecques et romaines de la place Jules-Verne à Marseille, CRAI, avril-juin, p. 459-484.

1995 Bouches-du-Rhône. Au large de Fos-sur-Mer, Martigues et Marseille. Dendrochronologie et dendromorphologie des épaves antiques de Méditerranée, dans Bilan scientifique DRASM 1994, Paris, Ministère de la Culture, p. 35.

1996 Nouvelles recherches sur les épaves antiques de Méditerranée : dendrochronologie et dendromorphologie, dans A. Demaison (dir), Pour qui la Méditerranée au $21^{e}$ siècle ? Navigation, échanges et environnement en Méditerranée, Actes du colloque, Montpellier, Okeanos, Le Corum, 1996, Montpellier, Maison de l'Environnement de Montpellier, p. 41-50 (avec F. Guibal).

1996 Var. Au large de Ramatuelle, La Croix-Valmer, Le Lavandou. Dendrochronologie et dendromorphologie des épaves antiques de Méditerranée, dans Bilan scientifique DRASM 1995, Paris, Ministère de la Culture, p. 56-57.

1997 Un exemple d'évolution des techniques de construction navale antique : de l'assemblage par ligatures à l'assemblage par tenons et mortaises, dans D. Garcia, D. Meeks (dir.), Techniques et économie antiques et médiévales. Le temps de l'innovation, Actes du Colloque international, Aix-en-Provence, 1996, Paris, Errance (Travaux du Centre Camille-Jullian, 21), p. 195-203.

1997 L'art de la navigation dans l'Antiquité, dans J. Leclant (dir.), Regards sur la Méditerranée, Actes du 7 Colloque de la villa Kérylos, Beaulieu-sur-Mer, 1996, Paris, AIBL (Cahiers de la villa « Kérylos », 7), p. 89-101.

1997 Var. Au large de Saint-Raphaël. Dendrochronologie et dendromorphologie des épaves antiques de Méditerranée, dans Bilan 
scientifique DRASSM 1996, Paris, Ministère de la Culture et de la Communication, p. 102-103 (avec F. Guibal).

Conception et réalisation des navires de l'Antiquité méditerranéenne, dans É. Rieth (dir.), Concevoir et construire les navires. De la trière au picoteux, Ramonville Saint-Agne, Éditions Erès, p. 49-72.

1998 Les épaves grecques du $\mathrm{VI}^{\mathrm{e}}$ siècle av. J.-C. de la place Jules-Verne à Marseille, dans P. Pomey, É. Rieth (dir.), Actes du 7e International Symposium on Boat and Ship Archaeology (ISBSA 7), Île Tatihou, 1994, Paris, CNRS Éditions (Archaeonautica, 14) p. 147154.

1998 L'utilisation du matériau-bois dans la construction navale antique d'après l'analyse anatomique et dendrochronologique : résultats préliminaires, dans É. Rieth (dir.), Méditerranée antique : pêche, commerce, navigation, Actes du Congrès National des Sociétés Historiques et Scientifiques, $120^{e}$, Aixen-Provence, 1995, 121'e Nice, 1996, Paris, CTHS, p. 159-175 (avec F. Guibal).

1998 Dendrocronologie et dendromorphologie, dans G. Volpe (dir.), Archeologia Subacquea, VIII Ciclo di lezioni sulla ricerca applicata in archeologia, Certosa di Pontignano (Siena), 1996, Florence, All'Insegna del Giglio, p. 425-446 (avec F. Guibal).

1999 Essences et qualité des billes employées dans la construction navale antique : étude anatomique et dendrochronologique, dans A. Corvol (dir.), Forêt et Marine. Groupe d'Histoire des Forêts Françaises, Paris, L'Harmattan, p. 15-32 (avec F. Guibal).

1999 Les épaves grecques archaïques de la place Jules-Verne ; l'activité des chantiers navals des témoignages précieux, dans A. Hesnard, M. Moliner, F. Conche, M. Bouiron (dir.), Parcours de villes, Marseille : 10 ans d'archéologie, 2600 ans d'Histoire, Aix-en-Provence, Musées de Marseille, Édisud, p. 35-36.

1999 Les épaves romaines de la place Jules-Verne à Marseille : des bateaux dragues?, dans H. Tzalas (ed.), Tropis V, Proceedings of the $5^{\text {th }}$ International Symposium on Ship Construction in Antiquity, Nauplia, 1993, Athènes, Hellenic Institute for the Preservation of Nautical Tradition, p. 321-328.

1999 Le port antique et ses deux épaves, dans A. Hermary, A. Hesnard, H. Tréziny (dir.), Marseille grecque. La cité phocéenne (600-49 av. J.-C.), Paris, Éditions Errance (Hauts lieux de l'histoire), p. $45-49$.

1999 Les bateaux grecs archaïques de Marseille, dans Les bateaux. De la trière aux voiles de courses, Luçon, Bibliothèque Pour La Science, p. 6-13.

2000 Un témoignage récent sur la pêche au corail à Marseille à l'époque archaïque, dans J.-P. Morel, C. Rondi-Costanzo, D. Ugolini (dir.), Corallo di ieri, corallo di oggi, Atti del Convegno, Ravello, 1996 , Bari, Edipuglia (Scienze e materiali del patrimonio culturale, 5), p. 37-39.

2000 Dendrochronologie et dendromorphologie des épaves antiques de Méditerranée (Bouches-du-Rhône et Var), Gallia Informations, 1998-1999, Paris, Éditions du CNRS (avec F. Guibal)

2001 Les épaves grecques archaïques du $\mathrm{VI}^{\mathrm{e}}$ siècle av. J.-C. de Marseille : épaves Jules-Verne 7 et 9 et César 1, dans H. Tzalas (ed.), Tropis VI, Proceedings of the $6^{\text {th }}$ International Symposium on Ship Construction in Antiquity, Lamia, 1996, Athènes, Hellenic Institute for the Preservation of Nautical Tradition, p. $425-437$.

2001 La trirème antique de Napoléon III : un essai d'archéologie navale expérimentale sous le Second Empire, dans Napoléon III et l'archéologie. Une politique archéologique nationale sous le Second Empire, Actes du Colloque, Compiègne, 2000, Compiègne, Société historique de Compiègne (Bulletin de la Société Historique de Compiègne), p. 239-266

2001 Préface, dans A. Cartault, La trière athénienne. Étude d'archéologie navale, Paris, Claude Tchou (Bibliothèque des Introuvables), p. 9-18.

2001 Le renouveau d'une discipline : historiographie de l'archéologie navale antique, dans J.-P. Brun, Ph. Jockey (éd.), Teknai. Techniques et sociétés en Méditerranée, Hommage à MarieClaude Amouretti, Paris, Maisonneuve \& Larose (L'atelier méditerranéen), p. 613-623.
Une nouvelle technique d'assemblage antique : l'assemblage de la membrure par ligatures et chevilles, dans $\mathrm{H}$. Tzalas (ed.), Tropis VII, Proceedings of the $7^{\text {th }}$ International Symposium on Ship Construction in Antiquity, Pylos, 1999, Athènes, Hellenic Institute for the Preservation of Nautical Tradition, p. 597-604.

2002 Bilan des recherches d'archéologie navale antique en France au cours du XX ${ }^{\mathrm{e}}$ siècle, dans H. Tzalas (ed.), Tropis VII, Proceedings of the $7^{\text {th }}$ International Symposium on Ship Construction in Antiquity, Pylos, 1999, Athènes, Hellenic Institute for the Preservation of Nautical Tradition, p. 893-901.

2002 Remarque sur la faiblesse des quilles des navires antiques à retour de galbord, dans L. Rivet, M. Sciallano (dir.), Vivre, produire et échanger : reflets méditerranéens. Mélanges offerts à Bernard Liou, Montagnac, Éditions Monique Mergoil (Archéologie et Histoire Romaine, 8), p. 11-19.

2002 Corse-du-Sud. Au large de Bonifacio. Dendrochronologie et dendromorphologie des épaves antiques de Méditerranée, dans Bilan scientifique DRASSM 2001, Paris, Ministère de la Culture et de la Communication, p. 85-86 (avec F. Guibal).

2002 Les graffiti navals de l'Alcazar à Marseille : des pentécontores phocéennes ?, dans H. Tzalas (ed.), Tropis VIII, Proceedings of the $8^{\text {th }}$ International Symposium on Ship Construction in Antiquity, Hydra, 2002, Athens, Hellenic Institute for the Preservation of Nautical Tradition [non publié].

2003 Timber Supply and Ancient Naval Architecture, dans C. Beltrame (ed.), Boats, Ships and Shipyards, Proceedings of the Ninth International Symposium on Boat and Ship Archaeology (ISBSA 9), Venice, 2000, Oxford, Oxbow books, p. 35-41 (avec F. Guibal).

2003 Reconstructing of Marseilles VI ${ }^{\text {th }}$ century BC Greek ships, dans C. Beltrame (dir.), Boats, Ships and Shipyards, Proceedings of the Ninth International Symposium on Boat and Ship Archaeology (ISBSA 9), Venice, 2000, Oxford, Oxbow books, p. 57-65.

2003 Var. Au large de Saint-Raphaël. Programme thématique : «Dendrochronologie et dendromorphologie des épaves antiques de Méditerranée », dans Bilan scientifique DRASSM 1998, Paris, Ministère de la Culture et de la Communication, p. 51-53 (avec F. Guibal).

2004 The Greek Sewn Shipbuilding Tradition and the Ma'agan Mikhael Ship: a comparison with Mediterranean parallels from the sixth to the fourth centuries BC, The Mariner's Mirror, 90, 1, p. 6-28 (avec Y. Kahanov).

2004 Principles and Methods of Construction in Ancient Naval Architecture, dans F. M. Hocker, C.A. Ward (eds), The Philosophy of Shipbuilding. Conceptual Approaches to the Study of Wooden Ships, College Station, Texas A\&M University Press, p. 25-36.

2004 Les navires du port grec archaïque de Marseille, Archaeologia Maritima Mediterranea, 1, p. 187-191 (avec A. Hesnard).

2004 Dendrochronologie et construction navale antique, Revue d'Archéométrie, 28, p. 35-42 (avec F. Guibal).

2004 Alpes-Maritimes. Au large de Cannes. Dendrochronologie et dendromorphologie des épaves antiques de Méditerranée : l'épave de la Tradelière, dans Bilan scientifique DRASSM 1999, Paris, Ministère de la Culture et de la Communication, p. 66-67 (avec F. Guibal).

2005 L'architecture navale grecque du $6^{\mathrm{e}}$ au $4^{\mathrm{e}}$ siècle av. J.-C, dans M. Egloff, D. Ramseyer (dir.), Amphore à la mer ! Épaves grecques et étrusques, catalogue de l'exposition, Hauterive, Laténium, Musée d'archéologie de Neuchâtel, p. 32-39.

2005 Archimede e la Syrakosia, dans E. Lo Sardo (dir.), Eureka! Il genio degli antichi, catalogue de l'exposition, Naples, Electa, p. 228-232 (avec A. Tchernia).

2005 Notices dans M.-P. Rothé, H. Tréziny (dir.), Marseille et ses alentours, Paris, AIBL (CAG 13/3), p. 364-369, 376, 413, 548549.

2005 Un nouveau témoignage sur la voile latine : la mosaïque de Kelenderis (v. 500 ap. J.-C., Turquie), dans H. Tzalas (ed.), Tropis IX, Proceedings of the $9^{\text {th }}$ International Symposium on Ship Construction in Antiquity, Agia Napa (Cypre), 2005, 
Athènes, Hellenic Institute for the Preservation of Nautical Tradition [non publié].

2006 The Kelenderis ship: A lateen sail, IJNA, 35.2, p. 326-329.

2006 Le rôle du dessin dans la conception des navires antiques. À propos de deux textes akkadiens, dans B. Mathieu, D. Meeks, M. Wissa (éds.), L'apport de l'Égypte à l'histoire des techniques. Méthodes, chronologie et comparaisons, Le Caire, IFAO (Bibliothèque d'étude, 142), p. 239-251.

2006 L'archéologie sous-marine et le bateau antique, dans A. Barkaoui, E. Rieth (éds.), Bateaux et ports méditerranéens. Bilan et perspectives, Actes de la table ronde internationale, Sfax-Kerkenna, 2005, Sfax, Université de Sfax, p. 65-88.

2006 Les graffiti navals de l'agorà. Étude préliminaire, dans M. Taslialan, T. Drew-Bear et al. (dir.), Fouilles de l'agora de Smyrne : rapport sur la campagne de 2005, Anatolia Antiqua, XIV, p. 327-337.

2006 Les navires étrusques : mythe ou réalité ? dans Gli Etruschi da Genova ad Ampurias. Atti del XXIV Convegno di Studi Etruschi ed Italici. Marseille-Lattes, 2002, Pise-Rome, Istituti editoriali e poligrafici internazionali, p. 423-434.

2006 Navires et construction navale, dans Marseille, de la grotte Cosquer à la grande peste, 27000 ans d'histoire, Archéologia, 435, juillet-août, p. 49-51.

2006 New light on the false clinkers in ancient Mediterranean shipbuilding, dans L. Blue, F. Hocker, A. Englert (eds), Connected by the sea, Proceedings of the Tenth International Symposium on Boat and Ship Archaeology (ISBSA 10), Roskilde, 2003, Oxford, Oxbow Books, p. 74-77.

2006 The Kelenderis Ship: A Lateen Sail, IJNA 35, 2, p. 326-329.

2006 L'archéologie navale. À propos de concepts et de méthodes, Archaeologia Maritima Mediterranea, 3, [2007], p. 165-172 (avec É. Rieth).

2006 Le rôle des maquettes dans les études d'archéologie navale, dans Archéologie Sous-Marine et Musée, Journée d'étude de l'Association Générale des Conservateurs des collections publiques de France, Provence-Alpes-Côte d'Azur, Arles, 2006, Lyon, AGCCPF-PACA, p. 63-68 (avec R. Roman).

2006 Les inventions entre l'anonymat et l'exploit : le pressoir à vis et la Syracusia, dans E. Lo Cascio (dir.), Atti del Convegno Innovazione tecnica e progresso economico nel mondo romano, Atti Incontri Capresi, Capri, 2003, Bari, Edipuglia, p. 81-99 (avec A. Tchernia).

2007 Les fouilles subaquatiques, sous-marines et en milieu humide. Historique et développements récents, dans H. Bernard-Maugiron, Ph. Coeuré, M. Clermont-Joly, J. Duchêne, P. Vaudaine, P. Veysseyre (éds.), Sauvé des eaux. Le patrimoine archéologique en bois. Histoire de fouilles et de restaurations, Grenoble, ArcNucléart, p. 15-21.

2007 Marseille, son port antique et les épaves de la place Jules-Verne, dans H. Bernard-Maugiron, Ph. Coeuré, M. Clermont-Joly, J. Duchêne, P. Vaudaine, P. Veysseyre (éds.), Sauvé des eaux. Le patrimoine archéologique en bois. Histoire de fouilles et de restaurations, Grenoble, Arc-Nucléart, p. 73-76 (avec A. Hesnard).

2008 Bernard Liou (1931-2006) : fondateur d'Archaeonautica, Archaeonautica 15, [2009], p. 9-12.

2008 J. Richard Dick Steffy and Ship and Boat Archaeology, dans $\mathrm{H}$. Tzalas (ed.), Tropis X, Proceedings of the $10^{\text {th }}$ International Symposium on Ship Construction in Antiquity, Hydra, 2008, Athènes, Hellenic Institute for the Preservation of Nautical Tradition [non publié].

2008 L'épave Jules-Verne 8 et autres données concernant l'activité des chantiers de construction navale antiques de Marseille, dans H. Tzalas (ed.), Tropis X, Proceedings of the $10^{\text {th }}$ International Symposium on Ship Construction in Antiquity, Hydra, 2008, Athènes, Hellenic Institute for the Preservation of Nautical Tradition [non publié].

2008 Attempts of dating Ancient shipwreck hulls in the Western Mediterranean by dendrochronology, dans H. Tzalas (ed.), Tropis X, Proceedings of the $10^{\text {th }}$ International Symposium on Ship Construction in Antiquity, Hydra, 2008, Athènes, Hellenic
Institute for the Preservation of Nautical Tradition (avec F. Guibal), [non publié].

2009 Dimensions et tonnage, dans X. Nieto, M. Santos (dir.), El vaixell grec arcaic de Cala Sant Vicenç, Girona, MAC-CASC (Monografies del CASC, 7), p. 60-64

2009 Principes et méthodes de construction en architecture navale antique méditerranéenne. De la conception à la réalisation, dans X. Nieto, M. A. Cau (dir.), Arqueologia Nàutica Mediterrània, Girona, MAC-CASC (Monografies del CASC, 8), p. 337-342.

2009 Des bateaux cousus aux bateaux à tenons et mortaises. Un exemple d'évolution des techniques de construction navale, dans X. Nieto, M. A. Cau (dir.), Arqueologia Nàutica Mediterrània, Girona, MAC-CASC (Monografies del CASC, 8), p. 357-362.

2009 Archéologie navale et archéologie expérimentale : les modèles d'étude, les maquettes de restitution et les répliques navigantes, dans X. Nieto, M. A. Cau (dir.), Arqueologia Nàutica Mediterrània, Girona, MAC-CASC, p. 411-417 (Monografies del CASC, 8).

2009 On the use of design in Ancient Mediterranean ship construction, dans H. Nowacki, W. Lefèvre (eds), Creating Shapes in Civil and naval Architecture. A cross-Disciplinary Comparison, Leiden-Boston, Brill, p. 49-63.

2009 Reply to Lucien Basch, «Were the Hittites able to build a replica of an Egyptian ship according to their own drawings? ", dans H. Nowacki, W. Lefèvre (eds.), Creating Shapes in Civil and naval Architecture. A cross-Disciplinary Comparison, LeidenBoston, Brill, p. 71-72.

2009 Avant-propos, dans P. Pomey (dir.), Transferts technologiques en architecture navale méditerranéenne de l'Antiquité aux Temps modernes : identité technique et identité culturelle, Actes de la Table Ronde Internationale, Istanbul, 2007, Istanbul, IFEA (Varia Anatolica, XX), p. 7-9.

2009 De l'assemblage par ligatures à l'assemblage par tenons et mortaises. Introduction, dans P. Pomey (dir.), Transferts technologiques en architecture navale méditerranéenne de l'Antiquité aux Temps modernes : identité technique et identité culturelle, Actes de la Table Ronde Internationale, Istanbul, 2007, Istanbul, IFEA (Varia Anatolica, XX), p. 15-29.

2009 Transfert de technologie et identité culturelle. Introduction, dans P. Pomey (dir.), Transferts technologiques en architecture navale méditerranéenne de l'Antiquité aux Temps modernes: identité technique et identité culturelle, Actes de la Table Ronde Internationale, Istanbul, 2007, Istanbul, IFEA (Varia Anatolica, XX), p. 131-136.

2009 Vers un renouveau des études de nautique égyptienne, dans I. Régen, F. Servajean (éds.), «Verba manent », recueil d'études dédiées à Dimitri Meeks par ses collègues et amis, Montpellier, Université Paul-Valéry Montpellier III, p. 325-330.

2009 Ancient shipwrecks, naval architecture and dendrochronology in the western Mediterranean, dans R. Bockius (ed.), Between the seas. Tranfer and Exchange in Nautical Technology, Proceedings of the Eleventh International Symposium on Boat and Ship Archaeology (ISBSA 11), Mainz, 2006, Mayence, Verlag des RGZM (RGZM Tagungen, 3), p. 219-226 (avec F. Guibal).

2009 A new approach to Mediterranean nautical archaeology. Harbour, river and river-sea boats, dans R. Bockius (ed.), Between the seas. Tranfer and Exchange in Nautical Technology, Proceedings of the $11^{\text {th }}$ International Symposium on Boat and Ship Archaeology, Mainz, 2006, Mayence, Verlag des RGZM (RGZM Tagungen, 3), p. 267-280.

2009 L'influence des techniques navales sur le contexte économique de la Grèce à la fin de l'époque archaïque, dans M. Wissa (ed.), The Knowledge Economy and Technological Capabilities. Egypt, the Near East and the Mediterranean 2nd millennium B.C. -1st millennium A.D., Proceedings of a conference held at the Maison de la Chimie, Paris, 2005, Sabadell, Barcelona, Editorial Ausa (Aula Orientalis Supplementa 26).

2011 Defining a ship: architecture, function and human space, dans A. Catsambis, B. Ford, D.L. Hamilton (eds.), Oxford Handbook of Maritime Archaeology, Oxford-New-York, Oxford Maritime Press, p. 25-46. 

lerie gallo-romaine, dans G. Boetto, P. Pomey, A. Tchernia (éds.), Batellerie Gallo-Romaine, Pratiques régionales et influences maritimes méditerranéennes, Paris/Aix-en-Provence, Éditions Errance/Centre Camille Jullian (BiAMA, 9), p. 9-15. A Comparative Study of Sewn Boats from Mediterranean, the Red sea and the Indian Ocean. The Question of Gujarat, dans L. Varadarajan (ed.), Gujarat and the Sea, Vadodara, Darshak Itihas Nidhi, p. 133-146.

2011 Les conséquences de l'évolution des techniques de construction navale sur l'économie maritime, dans W. Harris, K. Iara (eds), Maritime Technology in the Ancient Economy: Ship-design and Navigation, Portsmouth, Rhode Island, JRA (JRA, Suppl. Series, 84), p. 39-55.

2011 Sur les eaux d'Alexandrie : des navires et des bateaux, dans I. Hairy (dir.), Du Nil à Alexandrie. Histoire d'eaux, catalogue de l'exposition, Musée d'archéologie de Neuchâtel, octobre 2009mai 2010, Alexandrie, Centre d'Études Alexandrines, p. 518537.

2011 Les bateaux d'Ayn Soukhna. Les plus vieux vestiges de navires de mer actuellement connus, dans Les bateaux et la navigation en Égypte ancienne, Égypte, Afrique \& Orient, 64, p. 3-12.

2012 Ship remains at Ayn Soukhna, dans P. Tallet, E. Mahfouz (eds), The Red Sea in Pharaonic Times. Recent Discoveries along the Red Sea Coast, Proceedings of the Colloquium, Cairo-Ayn Sukhna, 2009, Le Caire, IFAO (BdE, 155), p. 35-52.

2012 Transition from Shell to Skeleton in Ancient Mediterranean Ship-Construction: analysis, problems, and future research, IJNA, 41.2, p. 235-314 (avec Y. Kahanov et É. Rieth).

2012 The Pharaonic Ship Remains of Ayn Soukhna, dans N. Günsenin (ed.), Between Continents, Proceedings of the Twelfth International Symposium on Boat and Ship Archaeology (ISBSA 12), Istanbul, 2009, Istanbul, Yayinlari, p. 7-15.

2012 Les graffiti navals de la zone minière du Sud-Sinaï, dans P. Tallet (dir.), La Zone minière pharaonique du Sud-Sinaï, Catalogue complémentaire des inscriptions du Sinaï I, Le Caire, IFAO (MIFAO, 130), p. 279-296.

2012 Honor Frost : une vie under the Mediterranean, Archaeonautica, 17, p. 7-9.

2012 Le dossier de l'épave du Golo (Mariana, Haute-Corse), Archaeonautica, 17, p. 11-30.

2013 On the Transition from Shell to Skeleton, IJNA, 42.2, p. 434-438 (avec Y. Kahanov et É. Rieth).

2013 Les bateaux « cousus » de Méditerranée de l'époque archaïque et l'épave du Golo, dans Ph. Pergola, F. Lo Schiavo, M. Miletti (dir.), Les lingots peau-de-bœuf et la navigation en Méditerranée centrale, Actes du II Colloque International, Lucciana, Mariana, 2005, Ajaccio, A. Piazzolla, p. 157-164.

2014 Le projet Prôtis. Construction de la réplique navigante d'un bateau grec du VI ${ }^{\mathrm{e}}$ siècle av. J.-C., CRAI, fasc. III, juillet-octobre, p. 1333-1357.

2015 The Madrague de Giens Project in the Wake of the Excavation of the Byzantine Shipwreck at Yassiada, dans D.N. Carlson, J. Leidwanger, S.M. Kampbell (eds), Maritime Studies in the Wake of the Byzantine Shipwreck at Yassiada, Turkey, College Station, Texas A\&M University, p. 73-81.

Navires et construction navale dans l'Egypte ancienne, dans B. Argémi, P. Tallet (éds.), Entre Nil et mers. La navigation en Égypte ancienne, Actes des rencontres de Provence Égyptologie, Arles, 2014, Paris/Bruxelles, Université Paris-Sorbonne/Université libre de Bruxelles (Nehet. Revue numérique d'égyptologie, 3), p. 1-29.

2015 La batellerie nilotique gréco-romaine d'après la mosaïque de Palestrina, dans P. Pomey (éd.), La batellerie égyptienne. Archéologie, Histoire, Ethnographie, Alexandrie, Centre d'Études Alexandrines (Études Alexandrines, 34), p. 151-172.

2015 Bateaux du Nil en terre cuite pour des dieux et des hommes, dans P. Pomey (éd.), La batellerie égyptienne. Archéologie, Histoire, Ethnographie, Alexandrie, Centre d'Études Alexandrines (Études Alexandrines, 34), p. 179-199 (avec P. Ballet).

2016 Determining an Architectural Family and its Evolution: The Exemple of the Greek Tradition of Sewn Shipbuilding in the Ancient Mediterranean, Studies of Underwater Archaeology, 2, Beijing, National Centre of Cultural Heritage of China, p. 329340.

2016 Ancre (p. 80-82); Construction navale (p. 284-288); Navigation - Antiquité (p. 1086-1073) ; Navire, dans D. Albera, M. Crivello, M. Tozy (dir.), Dictionnaire de la Méditerranée, Arles, Actes Sud.

2016 Le projet Prôtis, archéologie expérimentale, la réplique navigante comme moyen d'étude et de valorisation du patrimoine, dans Musées... port(s) et mer(s) entre histoire et patrimoine, Journée d'étude, Marseille, 2014, Marseille, Ville de Marseille, p. 91-96.

2017 The Prôtis project (Marseilles, France). The construction of a sailing replica of an Archaic Greek boat, dans in J. Gawronsky, A. van Holk and J. Schokkenbroek (eds), Ships And Maritime Landscapes, Proceedings of the Thirteenth International Symposium on Boat and Ship Archaeology, (ISBSA 13), Amsterdam, 2012, Eelde, Barkhuis Publishing, p. 484-489.

2017 À propos de la voile latine : la mosaïque de Kelenderis et les Stereometrica (II, 48-49) d'Héron d'Alexandrie, Archaeonautica, 19, p. 9-25.

2017 The Greco-Massaliot Shipwrecks in the Place Jules-Verne in Marseilles and the Evolution of Greek Ship Construction from the Sixth to the Fourth Century BC, dans S. Bouffier, D. Garcia (eds.), Greek Marseille and Mediterranean Celtic Region, New York, Peter Lang Publishing, p. 263-279.

2017 Ships and Shipping, dans A. Naso (ed.), Etruscology, vol. 1, Boston/Berlin, de Gruyter, p. 371-389.

2017 The Greek Archaic Sewn Boats Tradition and its evolution toward the mortise-and-tenon joint system, dans M. C. Morozzo Della Rocca, F. Tiboni (eds), Atti del convegno, Proceedings, $2^{\circ}$ Convegno nazionale, Transire mare, Cultura navale e marittima, Genova, 2016, Gênes, GoWare, p. 225-230.

2017 The Prôtis Project: the Gyptis Sailing Trials, in J. Litwin (ed.), Baltic and beyond. Change and continuity in shipbuilding, Fourteenth International Symposium on Boat and Ship Archaeology (ISBSA 14), Gdańk, 2015, Gdansk, National Maritime Museum, p. 229-236 (avec P. Poveda).

2018 Gyptis: Sailing Replica of a 6th-century-BC Archaic Greek Sewn Boat, IJNA, 47.1, p. 45-56 (avec P. Poveda). 


\section{CARNET PHOTOGRAPHIQUE}

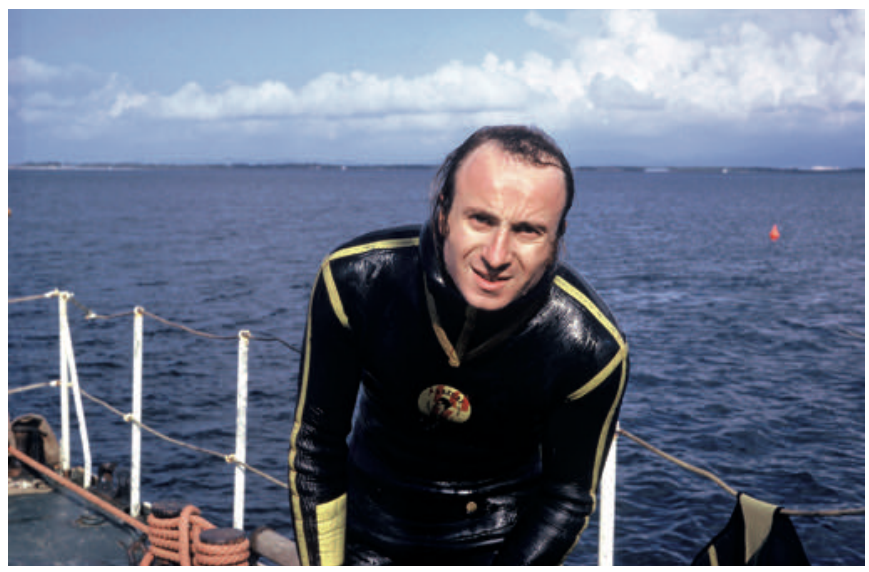

Fig. 1: Campagne de fouille de l'épave de la Madrague de Giens, (cliché A. Chéné, CNRS, CCJ, 1973.

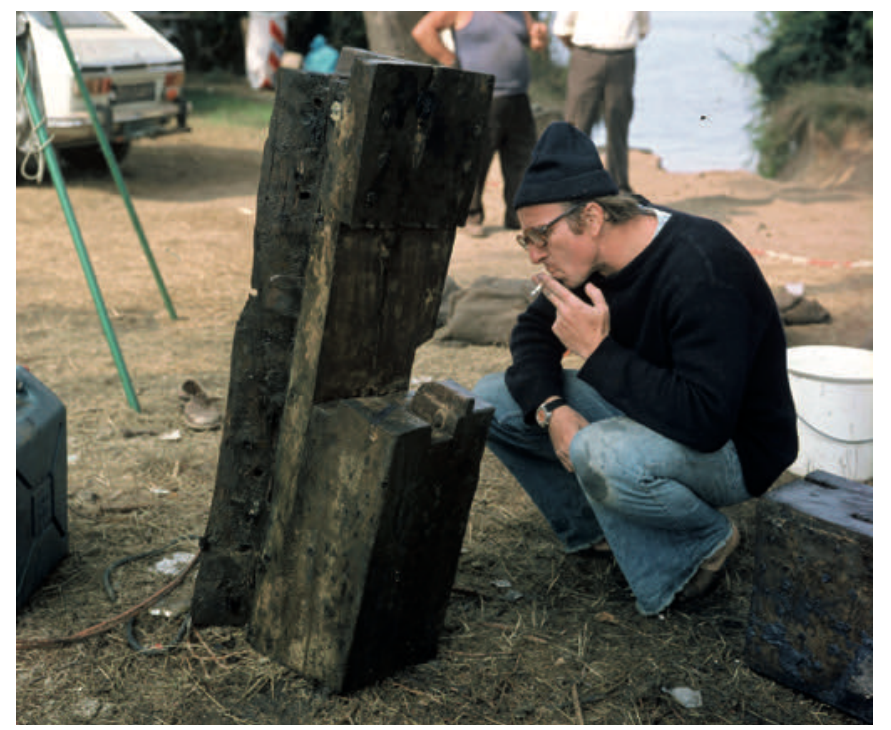

Fig. 2 : Patrice Pomey examine le prélèvement de la quille de l'épave de la Madrague de Giens (cliché A. Chéné, CNRS, CCJ, 1975).

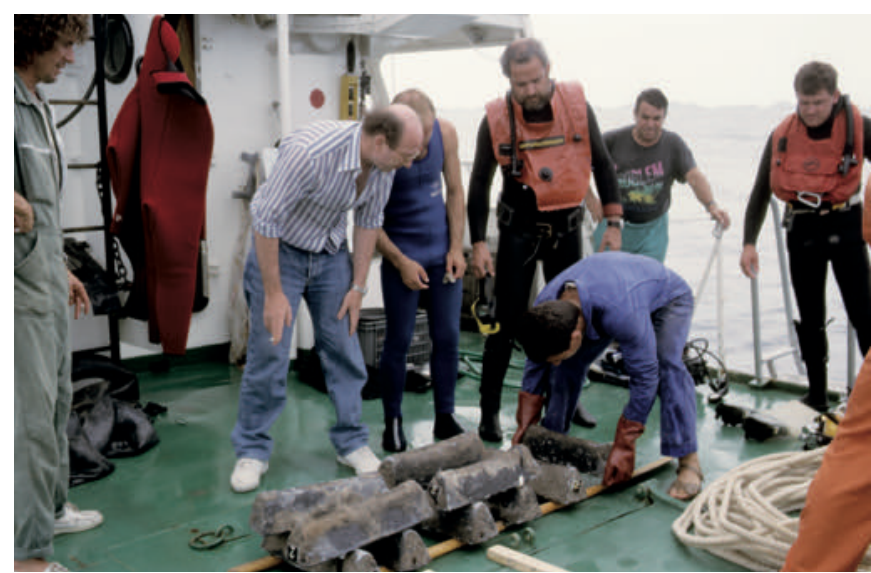

Fig. 4 : Avec Xavier Nieto sur le pont de L'Archéonaute, examinant les lingots de plomb remontés de l'épave Sud Perduto 2 en Corse (cliché A. Chéné, CNRS, CCJ, 1989). Bady, directeur du Patrimoine, dans la bibliothèque du Drassm au Fort SaintJean (cliché Guy Dauphin/Drassm, 1988).

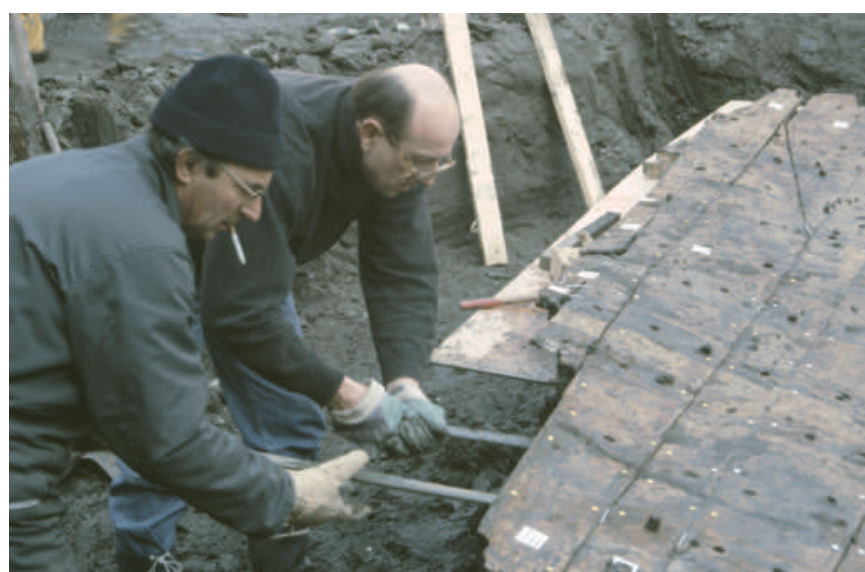

Fig. 5 : Avec Michel Rival lors des phases de récupération de l'épave JulesVerne 7 à Marseille (cliché Ph. Foliot, CNRS, CCJ, 1993).

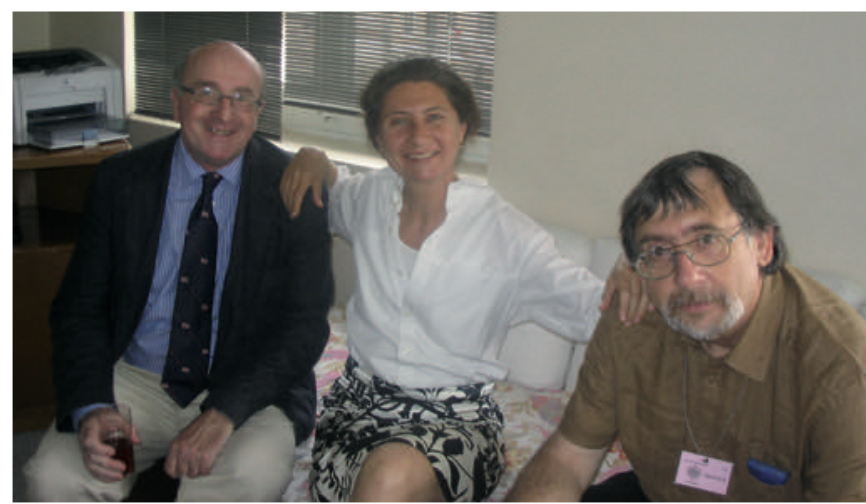

Fig. 6: Lors de la Table Ronde Internationale à l'Institut français d'Etudes Anatoliennes d'Istanbul avec Nergis Günsenin et Eric Rieth (cliché S. Marlier, 2007). 


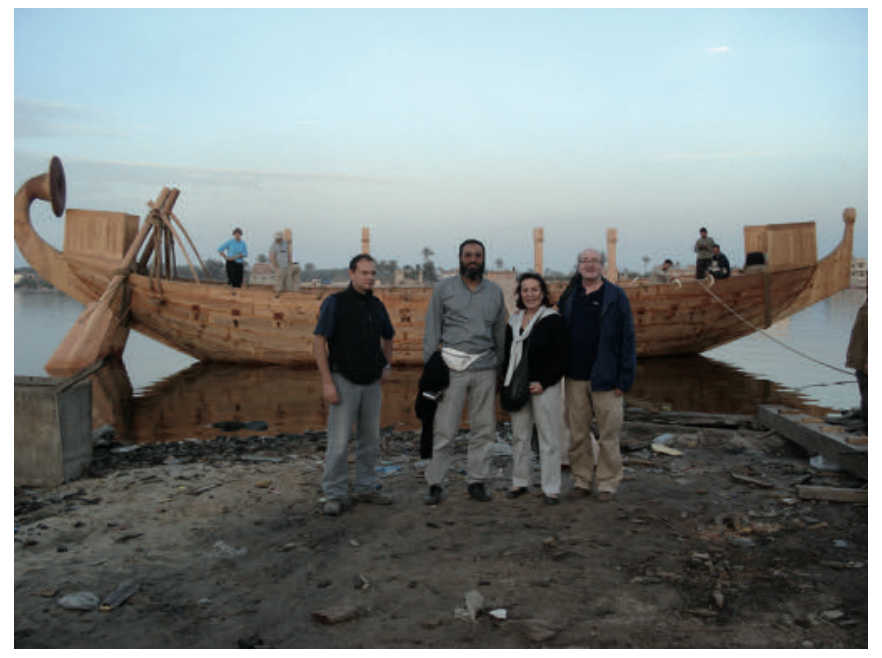

Fig. 7 : Avec Monique Pomey, Mohamed Abd el-Maguid et Laurent Borel sur le chantier Ebad El-Rahman à Rashid, Egypte, lieu de construction du Min du Désert (cliché A. Pelle, archives CE Alex/CNRS, 2010).

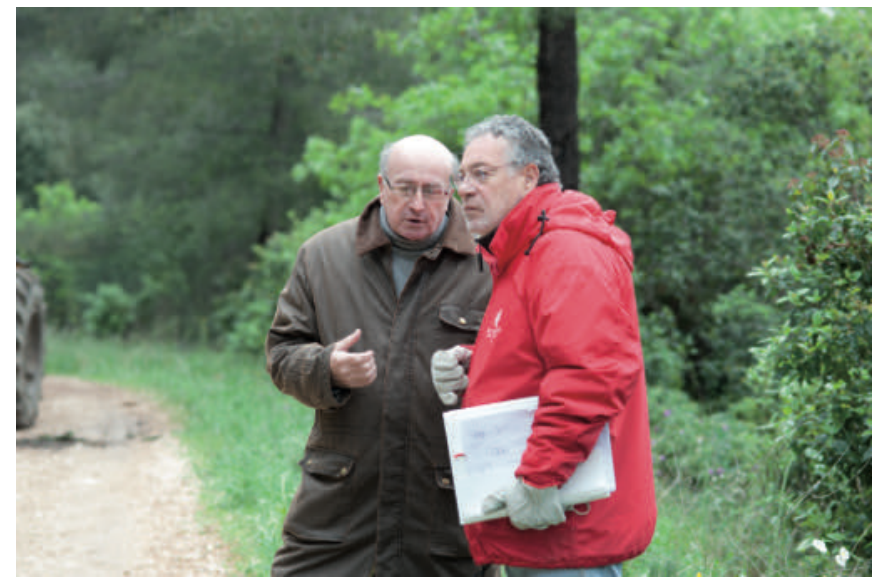

Fig. 9 : Choix des bois avec Robert Roman dans la forêt de Gémenos (cliché Ph. Groscaux, AMU, CNRS, CCJ, 2010).

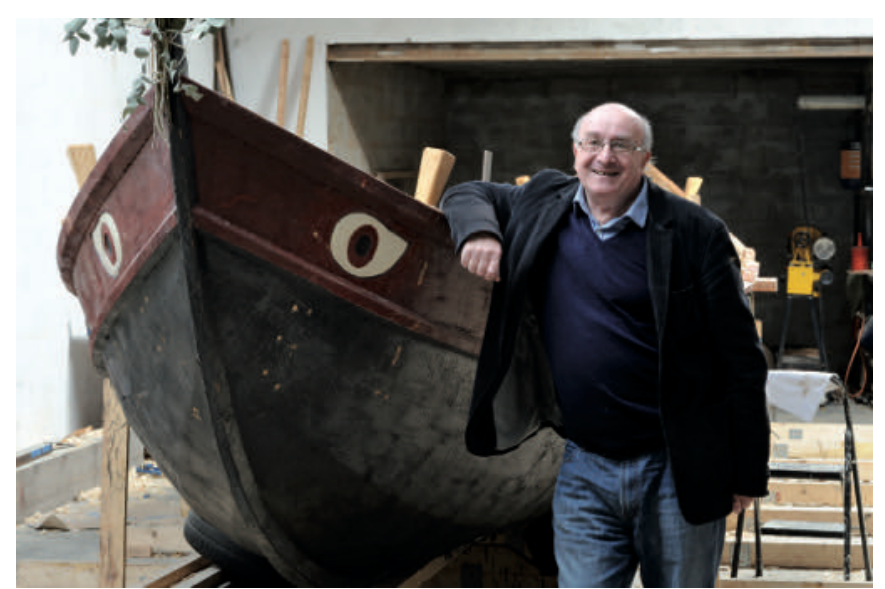

Fig. 11 : Avant le lancement du Gyptis (cliché L. Damelet, AMU, CNRS, CCJ, 2013).

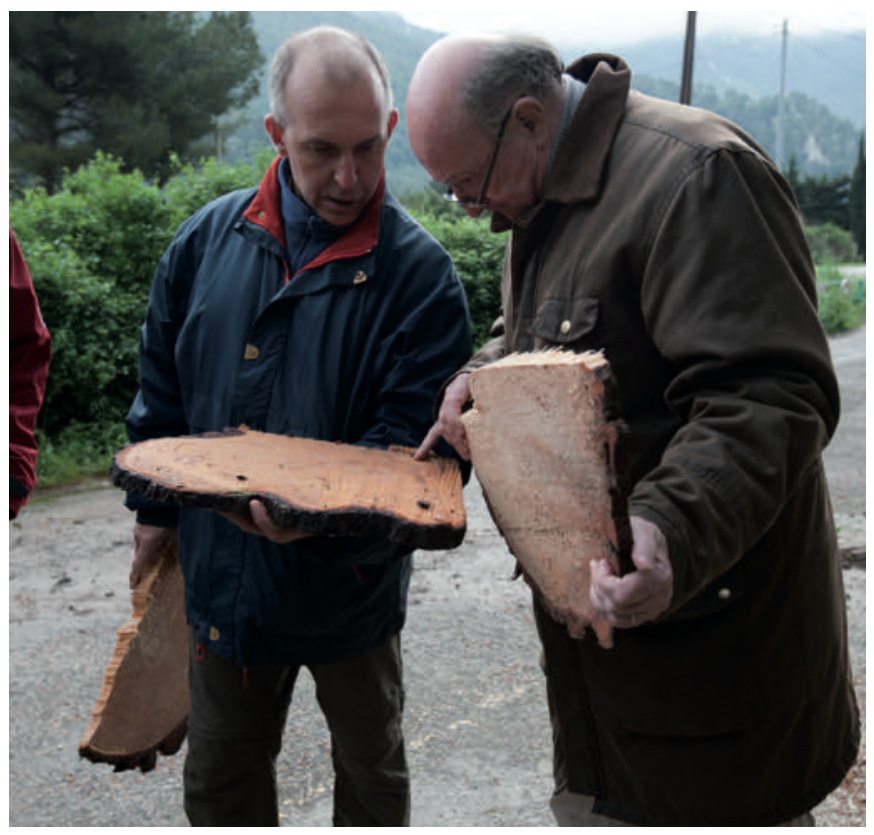

Fig. 8 : Examinant avec Frédéric Guibal les bois fraîchement coupés dans la forêt de Gémenos pour la construction du Gyptis (cliché Ph. Groscaux, AMU, CNRS, CCJ, 2010).

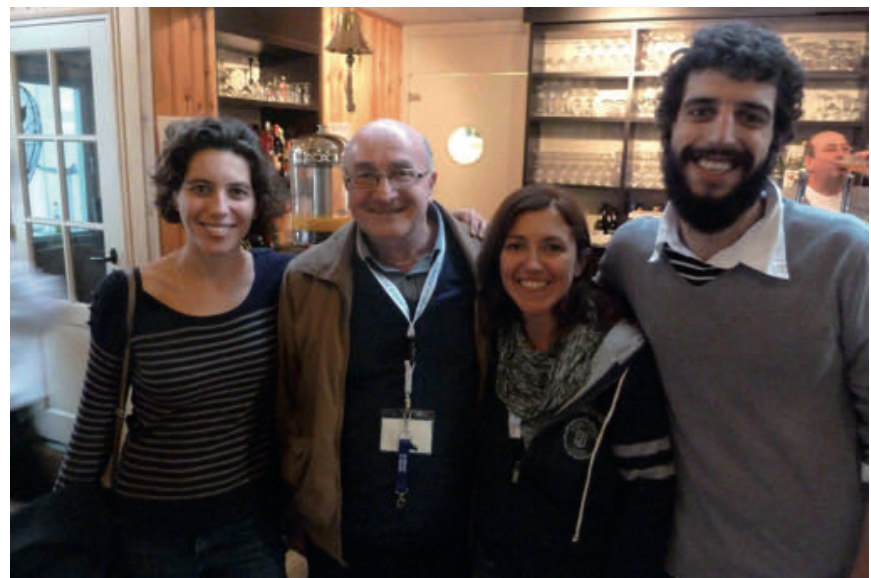

Fig. 10 : En 2012, avec ses élèves au 13e International Symposium on Boat and Ship Archaeology à Amsterdam : de gauche, Sabrina Marlier, Giulia Boetto et Pierre Poveda (cliché M. Pomey).

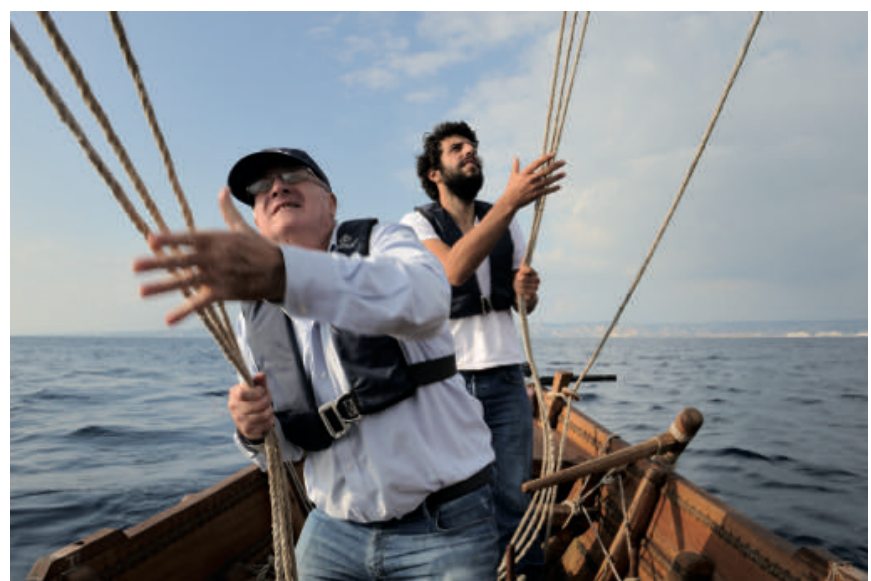

Fig. 12 : Avec Pierre Poveda à la manœuvre du Gyptis (cliché L. Damelet, AMU, CNRS, CCJ, 2015). 